\title{
A comparative analysis of two wind velocity retrieval techniques by using a single Doppler radar
}

\author{
H.-C. Lim $^{1}$ and D.-I. Lee ${ }^{2}$ \\ ${ }^{1}$ School of Mechanical Engineering, PuKyong National University, San 100, Yongdang-Dong, Nam-Gu, Busan, 608-739, \\ South Korea \\ ${ }^{2}$ Department of Environment and Atmospheric Science, PuKyong National University, 599-1, Daeyon-Dong, Nam-Gu, \\ Busan, 608-737, South Korea
}

Received: 18 January 2009 - Published in Hydrol. Earth Syst. Sci. Discuss.: 24 February 2009

Revised: 16 May 2009 - Accepted: 19 May 2009 - Published: 26 May 2009

\begin{abstract}
This study compares the theoretical basis of the two wind velocity retrieval methods, Velocity Azimuth Display (VAD) and Velocity Area Display (VARD) by using data obtained by a single Doppler radar. Two pre-assumed shapes of the wind velocity distribution with altitude are considered, uniform and parabolic. The former presents an approximation of the non-sheared or low-sheared wind flow in the upper troposphere, while the latter is a simplified representation of the Atmospheric Boundary Layer (ABL) in lower troposphere or high-sheared wind flow at the edges of the tropospheric jet streams. Both techniques for the wind velocity retrieval considered in this study are reformulated in order to get more precise information on the wind velocity components. An algorithm is proposed to decrease the uncertainty in retrieving by evaluating the coefficients of the polynomial equation and applying a transfer function with respect to the angle formed between the wind flow direction and direction of radar beam. It is concluded that, provided the formulated transformation functions are used, the application of the VAD and VARD techniques to the single-Doppler data may be an invaluable tool for solving various climate and wind engineering problems.
\end{abstract}

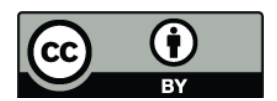

Correspondence to: D.-I. Lee

(leedi@ pknu.ac.kr)

\section{Introduction}

Changes in climate have had an increasing impact on wind environment of the Korean peninsula over the last decade. For example, several deadly typhoons such as Funsen and Rusa struck the Korean coast in 2002, while the typhoon Ewiniar, the 3rd largest in 2006, was particularly dreadful causing a great damage in a short period. Therefore, the importance of forecasting wind velocity features and maintaining an appropriate level of readiness and preparedness for similar disasters is beyond question. The forecast of wind parameters such as its speed, direction or convergence/divergence features, however, still remains a main concern of the meteorological services and the observatories.

One of the greatest advances in atmospheric remote sensing techniques is the application of the Doppler shift effect for development of the radar technology called Doppler radars. Doppler radars not only detect and measure the power received from a target, but also measure the target's velocity component in the direction in which the radar beam is pointed. When the target moves toward or away from the radar, the complete information concerning the target motion may be obtained. The target velocity in these directions is called radial velocity component, $V_{r}$. Because the Doppler radar may detect any kind of natural or artificial scatter moving in atmosphere with atmospheric wind, the application of Doppler radar technique for measuring vertical profiles of wind velocity or complete three-dimensional fields of air motion is very promising. It might significantly improve short-range forecasting of many dangerous meteorological phenomena such as microburst, thunderstorms and tornadoes. 


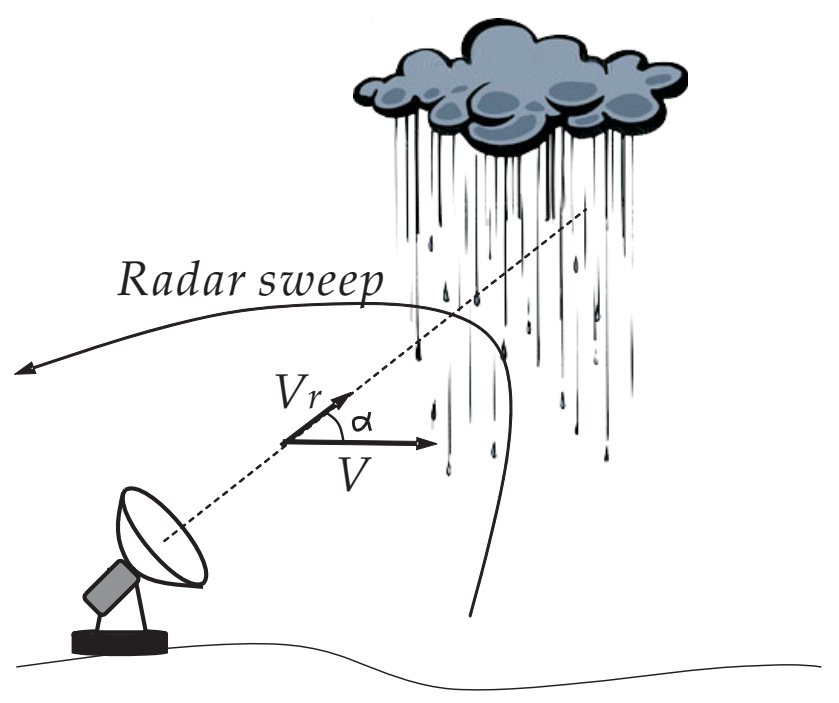

Fig. 1. A sketch showing the relationship between the radial velocity of the moving target, $V_{r}$, and real target velocity, $V$. Only the radial target velocity may be retrieved be a single Doppler radar without any additional assumptions. The angle $\alpha$ is formed between the direction in which the radar beam is pointed and direction in which target moves.

As described below, a number of radar studies have been carried out to obtain an appropriate three-dimensional distribution of the extreme wind fields in typhoons and heavy rainfall during summer monsoon season. Many developed countries spent a lot of time in trying to make an accurate retrieval of wind velocity fields from radars; whereas the studies on these retrieval methods in Korea were relatively out of primary concern. Lack of high performance radar, trained human resources and appropriate funding may be few reasons for relatively rare appearance of such kind of studies in Korea.

The antenna of the radar usually rotates around a vertical axis, scanning the horizon in all directions. Figure 1 shows the geometric relationship between the directions which a target (cloud or rain drops) moves and the direction in which antenna is pointed. A challenge is here to obtain information concerning the total target velocity, which may be carried out only with certain additional suppositions.

Lhermitte and Atlas (1961) were the first to demonstrate the crucial importance of the magnitude and direction of the mean horizontal wind velocity retrieved from radial velocity data. The proposed technique using the data processed from horizontal concentric circles centered at the radar site was called Velocity Azimuth Display (VAD). Caton (1963) and Browning and Wexler (1968) demonstrated that similar concept may be extended to yield other important parameters such as mean convergence, stretching and shearing deformation. They assumed that the wind vector field varies linearly in the horizontal plane. When using the VAD technique, it is not possible to separate the contributions of the vertical wind velocity and the hydrometer fall velocity to the total vertical velocity. Moreover, an additional problem is the divergence of the horizontal wind field. Improved versions of the VAD technique designed to properly separate various contributions were developed in the late eighties and early nineties and have been dubbed the extended-VAD (EVAD) technique (Srivastava et al., 1986) and the concurrent extended-VAD(CEVAD) technique (Matejka, 1993). Instead of processing a single VAD or a series of VADs, one can only process the low altitudes $\left(\phi \approx 0, z_{0} \approx 0\right)$ which are of greater interest than the other data. This method called Velocity ARea Display(VARD) was developed by Easterbrook (1975). His study was dealing with processing data in a conical sector rather than along a circle. Easterbrook showed that five parameters of the wind field may be extracted from such data; however, unless additional information is available, the mean horizontal velocity is contaminated by vorticity and cannot be completely determined. Nowadays, both the VAD and VARD techniques, as well as newly developed tools such as EVAD or Volume Velocity Processing (VVP) are widely used to retrieve wind profiles from Doppler radar observations.

Despite the widespread use of the VAD and VARD retrieval techniques, only a few verification or case studies involving the retrieved wind profiles have been reported so far. Moreover, evaluations between the VAD and VARD retrieval techniques applied to horizontal wind velocity data have been rarely published up to now. For example, Anderson (1998) published the results of verification study comparing VAD wind profiles with those obtained by using either radiosonde or numerical weather prediction involving the High Resolution Limited Area Model (HIRLAM). The actual wind in the atmosphere is fairly complicated, frequently changing significantly from one level to the next. Moreover, the magnitude of wind may changes over time and space. To describe such velocity fields accurately, the best approach is to make a simultaneous measurement by three Doppler radars. However, such measurement systems are fairly expensive and cover very limited area and thus rarely been accomplished. Most of the studies up to now were carried out by using reduced instrumental system involving two or one Doppler radar. We carry out some further analysis along this line and study the generalization of the wind field by processing the data from single-Doppler radar in volume, assuming linearity of the wind field distribution. The emphasis in this study is placed on a radar system for retrieval of wind velocity fields over Korea. In addition, we present some comparison of different implementation of the VAD and VARD retrieval techniques by using two shapes of the reference wind profiles, uniform and parabolic. 


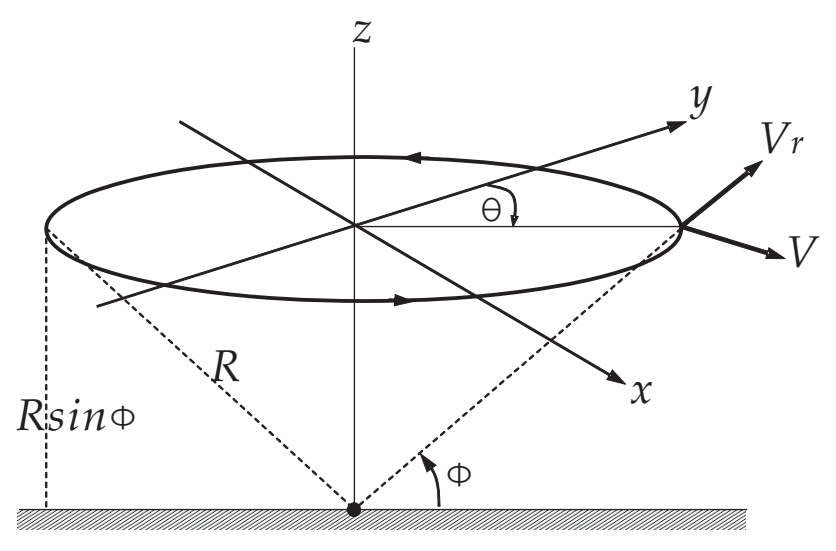

Fig. 2. Geometry of radar scan from Browning and Wexler (1968).

\section{Analysis of the mean wind field}

\subsection{Processing the velocity field}

As stated above, the success of the VAD and VARD techniques in predicting three-dimensional wind field heavily depends on the value of the angle formed between direction in which wind is blowing and direction in which antenna is pointed. Therefore, to analyze the role of all factors involved in application of those techniques, we reformulated those methods, i.e. VAD, VARD (Waldteufel and Corbin, 1978).

Consider a Cartesian coordinate system $(x, y, z)$ (see Fig. 2), whose origin 0 is at the radar, with vertical axis $z$ directed upward and axes $x$ and $y$ directed to the east and the north, respectively. The spherical coordinates $R, \phi$, and $\theta$ of the measured point are associated with the Cartesian system, where $R$ is the range, $\phi$ is the elevation angle, and $\theta$ is the azimuth angle starting from the north. Assume that the velocity vector of the motion of scatters $V=(u, v, w)$ varies linearly around its value $\left(u_{0}, v_{0}, w_{0}\right)$ at a point $\left(x_{0}, y_{0}, z_{0}\right)$ and may be represented as follows:

$$
\begin{aligned}
u & =u_{0}+\frac{d u}{d x}\left(x-x_{0}\right)+\frac{d u}{d y}\left(y-y_{0}\right)+\frac{d u}{d z}\left(z-z_{0}\right) \\
v & =v_{0}+\frac{d v}{d x}\left(x-x_{0}\right)+\frac{d v}{d y}\left(y-y_{0}\right)+\frac{d v}{d z}\left(z-z_{0}\right) \\
w & =w_{0}+\frac{d w}{d x}\left(x-x_{0}\right)+\frac{d w}{d y}\left(y-y_{0}\right)+\frac{d w}{d z}\left(z-z_{0}\right)
\end{aligned}
$$

Then the radial velocity $V_{r}$ measured by the radar may be written as follows,

$$
V_{r}=-u \cos \theta \cos \phi-v \sin \theta \cos \phi-w \sin \phi
$$

where $\theta$ and $\phi$ are the azimuth and elevation angles. Transforming from rectangular to polar coordinates, introducing the radial distance $R$ and regrouping terms, the following equation may be obtained

$$
\begin{aligned}
V_{r} \approx & \cos \theta \cos \phi u_{0}+\sin \theta \cos \phi v_{0}+\sin \phi w_{0} \\
& +R \cos ^{2} \theta \cos ^{2} \phi(d u / d x)+R \sin ^{2} \theta \cos ^{2} \phi(d v / d y) \\
& +R \sin \theta \cos \phi \cos ^{2} \phi(d u / d y+d v / d x) \\
& +\sin \phi\left(R \sin \phi-z_{0}\right) d w / d z \\
& +\cos \theta \cos \phi\left(R \sin \phi-z_{0}\right) d u / d z \\
& +\sin \theta \cos \phi\left(R \sin \phi-z_{0}\right) d v / d z
\end{aligned}
$$

In Eq. (3), only terms with different azimuth dependence can be discriminated. Since $R$ and $\phi$ are constant, we can regroup terms in Eq. (3) to obtain equation used in the VAD method.

$$
\begin{aligned}
V_{r} \approx & \cos \theta \cos \phi u_{0}+\sin \theta \cos \phi v_{0} \\
& +\cos ^{2} \theta\left[R \cos ^{2} \phi(d u / d x)+\sin \phi w_{0}\right] \\
& +\sin ^{2} \theta\left[R \cos ^{2} \phi(d v / d y)+\sin \phi w_{0}\right] \\
& +\sin \theta \cos \theta R \cos ^{2}(d u / d y+d v / d x)
\end{aligned}
$$

The VARD method proposed by Easterbrook (1975), is also applied for a conical sector, i.e., $x_{0} \neq 0, y_{0} \neq 0$. Therefore, when low elevation angles are considered $(\sin \phi \sim 0$, $\cos \phi \sim 1$ ), the Eq. (3) becomes

$$
\begin{aligned}
V_{r} \approx & \cos \theta\left(u_{0}-x_{0} d u / d x-y_{0} d u / d y\right) \\
& +R \sin \theta\left(v_{0}-x_{0} d v / d x-y_{0} d v / d y\right) \\
& +R \cos ^{2} \theta d u / d x+R \sin ^{2} \theta d v / d y \\
& +R \cos \theta \sin \theta(d u / d y+d v / d x)
\end{aligned}
$$

In Eq. (5), there are only terms regarding the horizontal plane so that it appears to be a two-dimensional linear equation. Given that the reference wind fields are provided, all derivatives as well as the trigonometric functions can be easily retrieved. Note here that the vorticity and mean horizontal wind components are combined in the first two terms and the differential properties of the horizontal wind field would be known to be restricted to low elevation angle (lower than $5^{\circ}$ ), as Easterbrook proposed. Therefore, the current calculation can confirm the vertical/horizontal velocity component varying with altitude even though the equation is restricted to low elevations.

\subsubsection{Reference wind field in a virtual pseudo-domain}

A uniform wind velocity profile modeling precipitation falling at terminal velocity and a parabolic wind velocity profile modeling typical wind profiles in atmospheric boundary layer will be used as the reference wind velocity distributions in this study. The case of uniform wind velocity distribution was already extensively studied by Lhermitte and Atlas (1961). They showed how the measurement of the velocity along concentric circles centered at the radar at a constant angle of elevation makes it possible to retrieve the vertical wind profile in terms of speed and direction. 

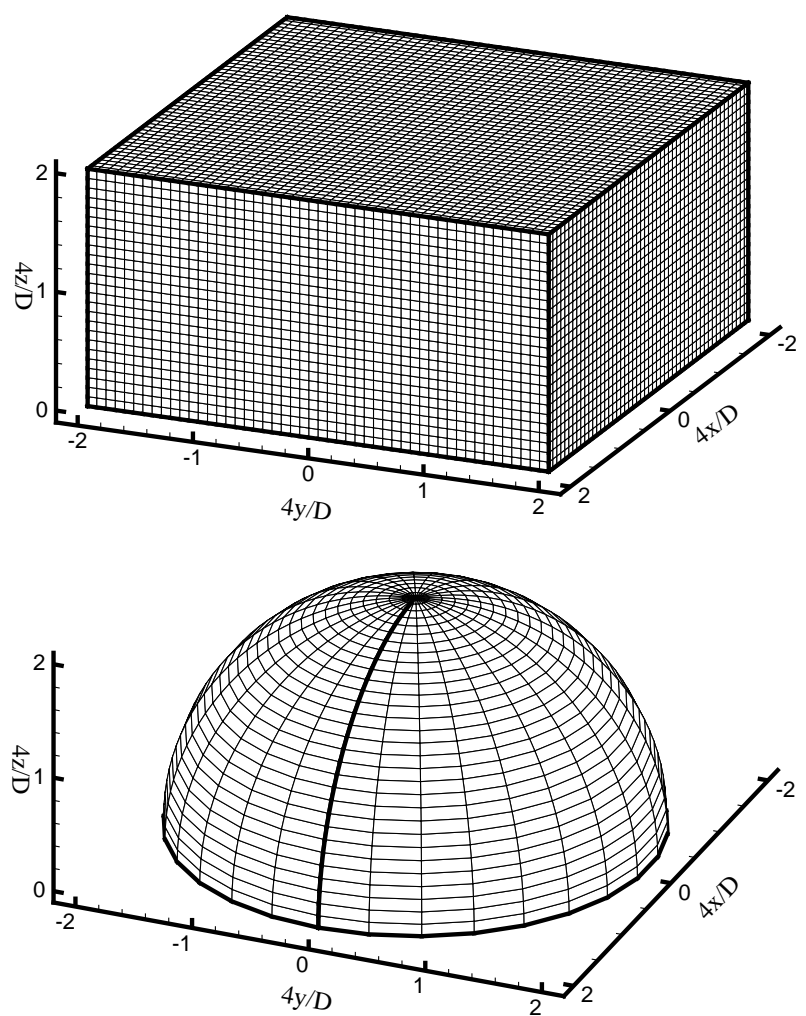

Fig. 3. Virtual domain for generating the wind profiles (a) in the rectangular coordinate (upper) (b) in the spherical coordinate (lower).

Figure 3 compares the size and shape of the virtual pseudo-domains obtained in Cartesian and spherical coordinate system. The size of the Cartesian coordinate system is $2^{\text {width }} \times 2^{\text {length }} \times 1^{\text {height }}$ non-dimensional units (i.e. $4 x / D, 4 y / D$ and $4 z / D$ ) and the numbers of grid are $50 \times 50 \times 25$. The size of the spherical system is 2 non-dimensional units, and the number of grid points are $30^{\text {radial }} \times 30^{\text {azimuth }} \times 30^{\text {elevation }}$. Each axis is nondimensionalized by $D / 4$, which is, in fact, the representative length of the pseudo-domain.

Figure 4 shows the reference wind velocity profiles allocated in the virtual domain. The case of the uniform wind flow (dashed line) with a constant velocity(e.g. 1) throughout the scanned layer is unrealistic, however, it is easy to deal with it. The parabolic or power-law profile (solid line) models the boundary layer with effect of surface roughness and, therefore, is closer to the real situation in the atmosphere. Comparing these two profiles, it is possible to analyze the effect of the atmospheric boundary layer on the air flow near to the ground. We refer to these particular velocity patterns as "wind signatures" according to the research by Armstrong and Donaldson (1969) and Wood and Brown (1986, 1997). Armstrong and Donaldson considered a series of concen-

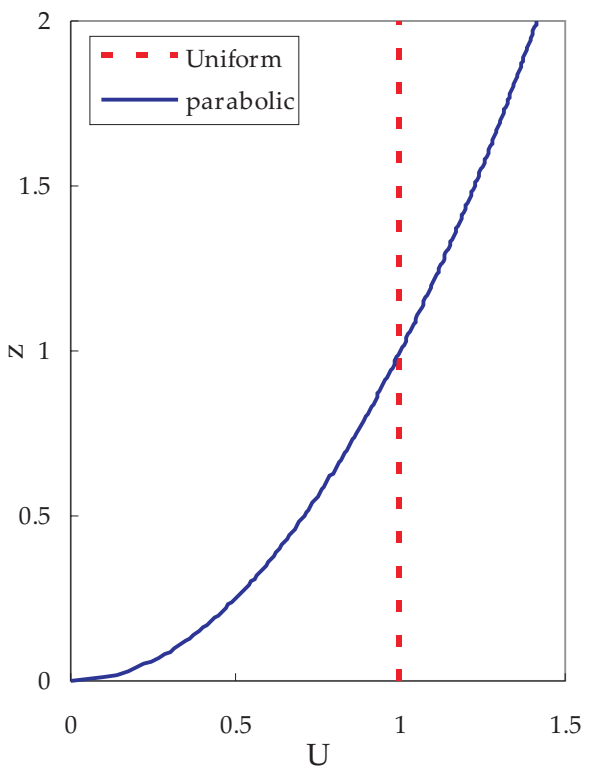

Fig. 4. Distributions of the streamwise wind velocity component, $U$, retrieved by using VAD method and two shapes of the reference wind velocity distribution: uniform (on the left-hand side) and parabolic (on the right-hand side).

tric arcs produced at a Plan Shear Indicator (PSI) display. This procedure allowed them to identify a small scale vortex, such as a meso-cyclone or tornado, from the observed tangential shear of the radial velocity. Wood and Brown produced computer-simulated pictures for various pre-assumed non-divergent wind velocity distributions. They used as a pattern the isopleths of constant Doppler velocity which they called isodops.

The typical procedure to retrieve the wind flow field includes: (i) generation of the virtual domain and allocation of the input environmental velocity; (ii) retrieval of the radial velocity by using VAD/VARD transformation; (iii) comparison of the real and the transformed velocities. More detail procedure will be explained in due course.

\section{Results and discussion}

Firstly, we present the calculated three-dimensional (3-D) distributions of the retrieved wind velocity components obtained by using both VAD and VARD techniques and by application of two proposed shapes of the reference wind velocity profiles. Then, a detailed analysis will be given of the calculated results. Finally, by using the mathematical techniques described below, it is possible to improve the application of the retrieved information from the Doppler radar and to get the shapes of retrieved wind velocity profiles closer to the pre-assumed shapes. 

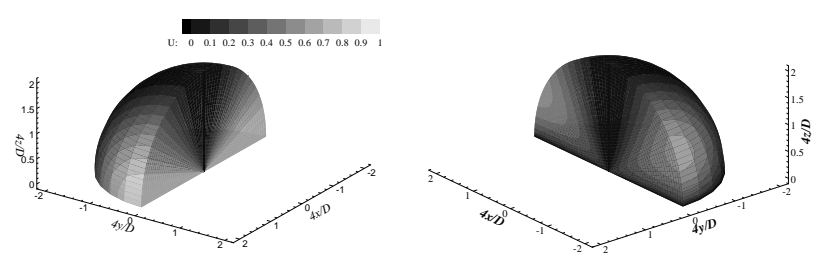

Fig. 5. Distributions of the streamwise wind velocity component, $U$, retrieved by using VAD method and two shapes of the reference wind velocity distribution: uniform (on the left-hand side) and parabolic (on the right-hand side).

\subsection{Analysis of the overall flow around the radar site}

In reality, the antenna of a scanning Doppler radar makes a rotation of $360^{\circ}$ per several seconds at a specific elevation, and then the scanning is continued at a new elevation. This procedure continues until the entire volume around the radar is scanned. The information on the scanned volume is obtained by superposition of the information from the radar beams at different elevation angles. For the sake of simplicity, the shape of beams is considered to be conical, while each of them may be divided into equi-spaced volumes containing information on the reflectivity of the target and the radial component of the wind velocity in the scanned volume. If the approximate shape of the wind velocity distribution with altitude in the environment is given, then both the streamwise and vertical components of the wind velocity may be derived. However, depending on the preassumed shape of the wind velocity profile, completely different results may be retrieved. In Fig. 5, the two calculation results are compared of the streamwise velocity component, both of them obtained by using the VAD method, but with different pre-assumed wind velocity distributions with altitude: uniform, $\mathbf{U}=(\mathrm{U}, 0,0)$, on the left-hand side, and parabolic, $\mathbf{U}=\left(\mathrm{az}^{2}, 0,0\right)$, on the right-hand side. The retrieved velocities are divided by the maximal value of preassumed velocity to obtain a non-dimensionalized field ranging from 0 to 1 . The contours of approximately equal values of non-dimensional velocity are drawn at every 0.1 unit, while the black-grey scale is used to distinguish the lower values (black) from the higher values (grey) of the nondimensional velocity. Thus, the grey-contoured fields designate the calculation domains where the retrieved values match pre-assumed ones perfectly, while the black-contoured fields designate the domains with poor coincidence. To clearly visualize the domains with different degree of matching inside the calculated volumes, the figure presents the cross-section of the calculated wind velocity fields. One may conclude from Fig. 5 that the degree of matching between the retrieved wind velocity fields and the pre-assumed ones is completely different in the case of uniform and parabolic pre-assumed wind velocity distributions. In the former case
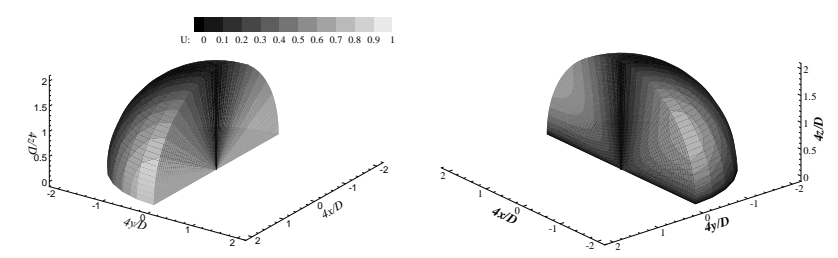

Fig. 6. The same as in Fig. 5, but using VARD method.

the best agreement is obtained near to the ground at low elevations when the radar is pointed in the directions either upstream or downstream to the blowing wind, i.e., westward or eastward, while in the latter case the agreement is observed in the same directions but at some height above the ground. Such result has been normally anticipated considering that the angle formed between the directions of the radar beam and blowing wind is minimal in such cases. As the elevation increases and/or azimuth changes from these two unique directions, the above mentioned angle increases and the possibility for correct evaluation of the real velocity drastically declines. When the radar is pointed vertically up or in the direction normal to the blowing wind, i.e. northward or southward, the retrieving of the wind velocity data is theoretically and practically impossible. In the case of a parabolic wind velocity distribution, the function of the non-dimensional retrieved wind velocity possesses well-expressed minimum near to the ground at all azimuths. This is only a consequence of the proposed way of non-dimensionalization. Since the preassumed velocity decreases significantly as one approaches to the ground, the division by maximum wind velocity produces misleading minimum near to the ground. As a result, the two cone-shaped zones of maximum of the nondimensional wind velocity are placed at some height above the ground in the directions upstream and downstream to the blowing wind, i.e., westward and eastward. The heights at which these maximums can be observed depend, in such a formulation, on the shape of parabolic pre-assumed wind velocity distribution.

Figure 6 presents the calculation results of the streamwise velocity component obtained by using the VARD technique for the same two pre-assumed wind velocity distributions with altitude. No significant difference is observed between Figs. 5 and 6 except that the uncertainty domain observed above the radar site when it is pointed vertically is significantly narrower in the case of application of VARD method as compared to the VAD method.

Consider now the fields of the vertical wind velocity obtained by using the VAD and VARD techniques by applying the two pre-assumed wind velocity distributions with altitude. The results presented in Figs. 7 and 8 correspond to the cases involving VAD and VARD techniques, respectively. 

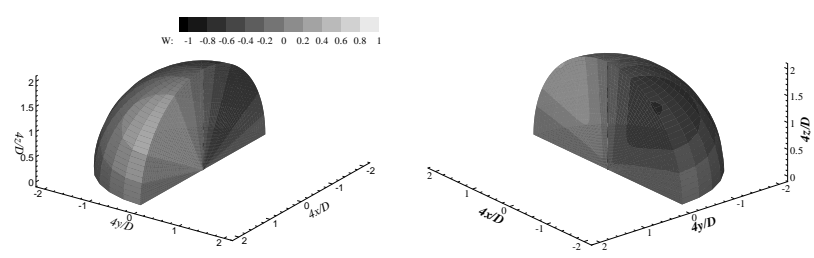

Fig. 7. Distributions of the vertical wind velocity component, $W$, retrieved by using VAD method and two shapes of the reference wind velocity distributions: uniform (on the left-hand side) and parabolic (on the right-hand side).
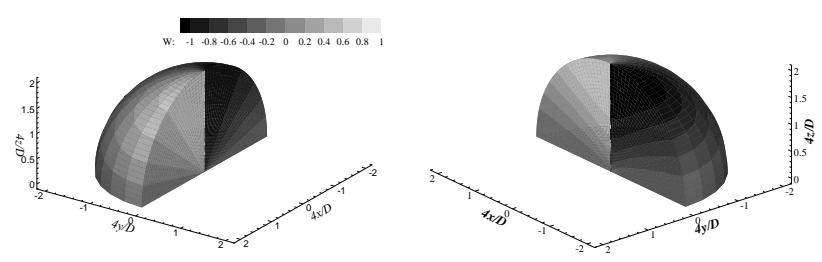

Fig. 8. The same as in Fig. 7, but using VARD method.

Figure 7 shows the rough distributions of the vertical wind velocity component, $W$, retrieved by using VAD method, whereas Fig. 8 by VARD method. The pre-assumed wind velocities are also applied in two different shapes - uniform (on the left-hand side) and parabolic (on the right-hand side).

The main difference in Figs. 7 and 8 is that strangely, there does not appear to be consistent in maximum region and considerable variation along a vertical line (i.e., the virtual central location of a radar) in case of the VARD method. As shown in the equation 5 , it would mainly consider the horizontal plane (i.e., due to considering a two-dimensional linear equation) and limited restriction of low elevation angle (lower than $5^{\circ}$ ) so that there is a big jump along the $4 x / D=0$ plane, which is not good enough to estimate whole vertical wind domain.

Figure 9 compares the streamwise velocity component on the surface of the hemisphere by using the VAD and VARD techniques and applying the above-mentioned reference wind velocity distributions. Consider the case of uniform reference wind velocity profile, presented in Fig. 9a. Comparing the results obtained by VAD and VARD techniques, one may conclude that in former case the stripes presenting an approximately constant value of the streamwise velocity component have a constant width everywhere on the surface of hemisphere, while in the latter case the stripes are significantly wider at low elevations, i.e. near to the ground, while become narrower at higher elevations. In particular, as the elevation angle $\phi$ approaches to goes to the $90^{\circ}$, the VARD-processed stripes of the constant streamwise velocity component become even narrower than the VAD-processed
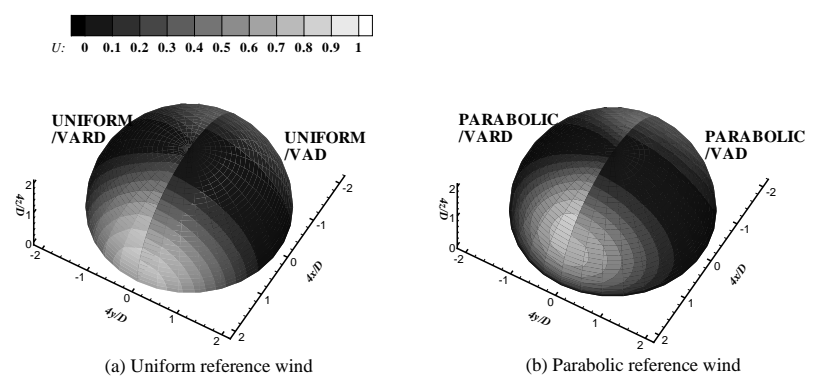

Fig. 9. Combined contour plots of the streamwise wind velocity component, $U$, on the surface of calculated domain varying as functions of elevation and azimuth for two reference wind velocity distributions: (a) uniform ; (b) parabolic. The calculated domain in both cases is divided into two halves showing the results obtained by applying VARD and VAD techniques, on the left-hand half and on the right-hand half, respectively.

ones. This difference is observed because the term involving elevation was ignored at low elevations in Eq. (5), which produced some errors at higher elevations.

In addition, Fig. $9 \mathrm{~b}$ considers the case of a parabolic reference wind velocity profile. Interestingly, the VARD method has the maximum peak region in higher elevation angle.

Proper quantitative assessment of the agreement between the analyzed and processed fields, however, requires a more detailed consideration of the specific wind profiles and curvefitting analysis. This will be covered in the next section.

\subsection{Details of the analysis}

We turn to consider several detailed flow scans at all azimuths at constant values of the elevation angle, $\phi$. Figure 10 presents the variation in the following transformed wind velocity components: $U$, streamwise (a), $V$, spanwise (b), and $\mathrm{W}$, vertical (c) together with variations of radial wind velocity, $V_{r}$ (d) as functions of azimuth obtained by application VAD technique to the uniform reference wind velocity distribution. Since all transformed wind velocity components are compared to the applied reference wind velocity distribution, the streamwise wind velocity component should be matched to the constant value 1 , while the crosswise and vertical ones should be matched to 0 . In addition, all of them, including radial wind velocity component, $V_{r}$, depends on the azimuth and elevation angle, $\theta$ and $\phi$, respectively. It may be seen from the figure that, as the elevation angle increases, the streamwise wind velocity component, $U$, as well as the radial velocity, $V_{r}$, decreases at all azimuth angles, $\theta$, except at those of around $90^{\circ}$ and $270^{\circ}$, where it remains at all times indefinite and equal to 0 (i.e., kind of pivot point). It is also worth mentioning that increasing the elevation angle noticeably reduces $U$ from the reference value of 1 at azimuth angles of 0 and 180 . This deflection is shown by 
(a)

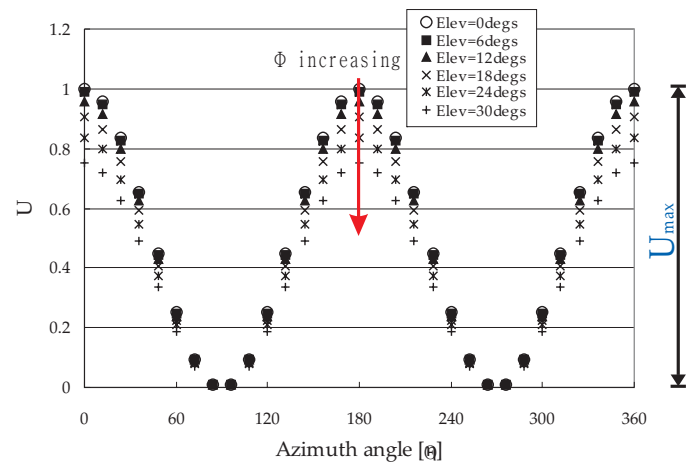

(b)

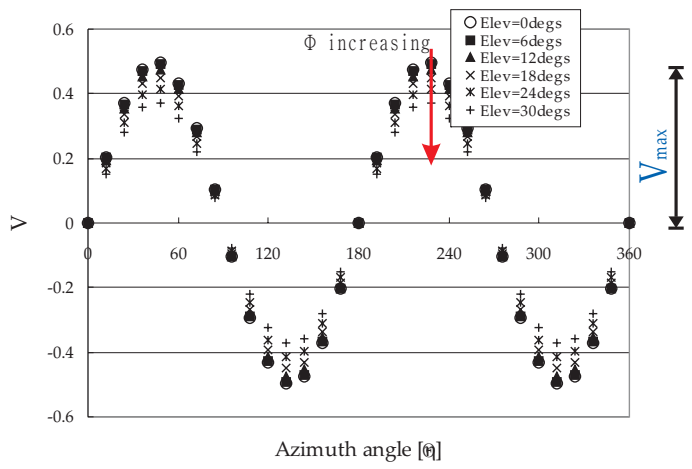

(c)

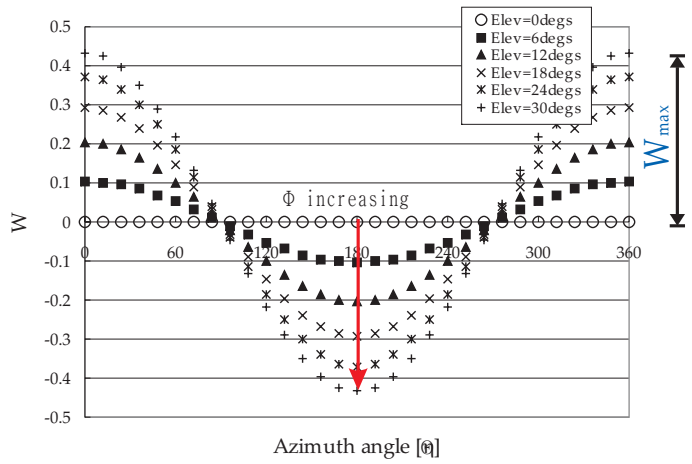

(d)

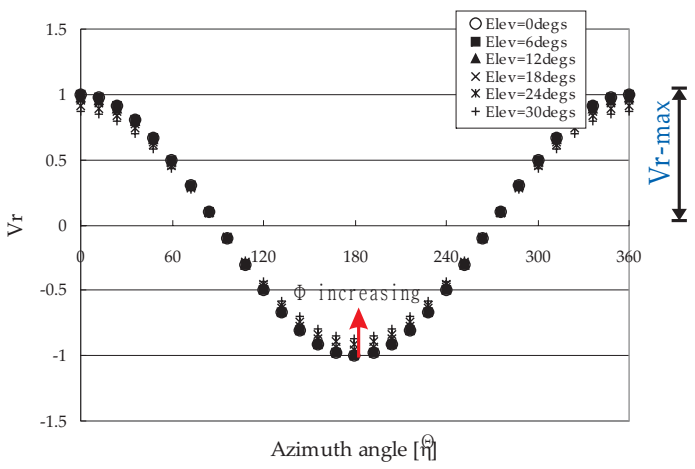

Fig. 10. Variations of the transformed velocity components $U$ (a), $V$ (b) and $W$ (c) and radial velocity component $V_{r}$ (d) obtained by using VAD technique as functions of the azimuth angle, $\theta$, at different elevation angles, $\phi$. The uniform wind velocity profile is taken as a reference wind velocity field.

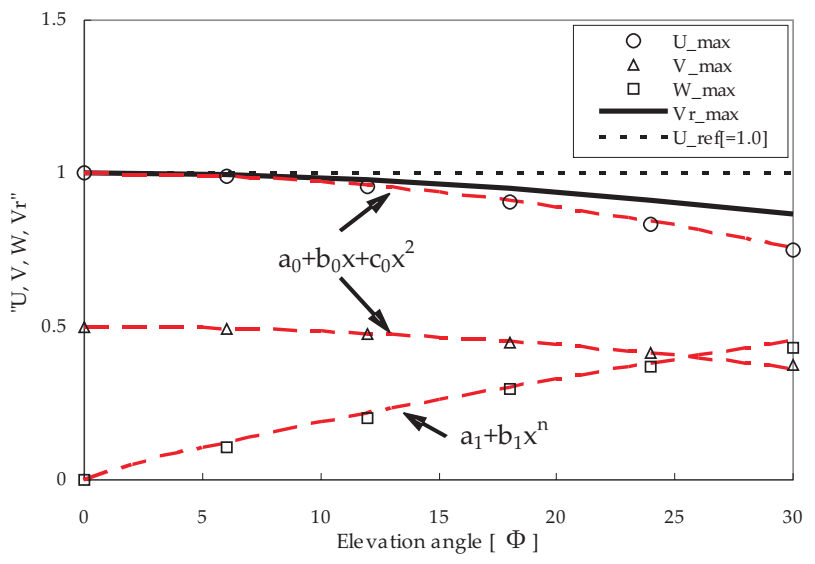

Fig. 11. Variations of maximums of the transformed velocity components $U$ (circles), $V$ (triangles), and $W$ (squires) and maximum of radial velocity component $V_{r}$ (solid line) as functions of the elevation angle $\phi$. The uniform wind velocity profile is taken as a reference wind velocity field, which is presented by short-dashed line. Long-dashed lines present polynomial and power-series fittings of the transformed velocity components.

the red arrow at the first diagram in Fig. 10. Conversely, the crosswise wind velocity component, $V$, approaches closer to the reference value of 0 at all azimuth angles as the elevation angle increases. These changes are shown by the red arrow at the second diagram in Fig. 10. The exceptions are two values of azimuth angles of $0^{\circ}$ and $180^{\circ}$, where the crosswise wind velocity component remains constant and equal to the reference value of 0 at all times. However, the greatest changes produced by changing the elevation angle are observed in the vertical wind velocity component, $W$. As the elevation angle increases, $\mathrm{W}$ progressively deflects from the reference value of 0 at all azimuth angles, except at those of $90^{\circ}$ and $270^{\circ}$. These changes are shown by the red arrow at the third diagram in Fig. 10.

Figure 11 presents a closer view of the variations of maximums of the calculated streamwise (circles), crosswise (triangles) and vertical (squares) wind velocity components observed at azimuth angles of $0^{\circ}, 45^{\circ}$ and $0^{\circ}$, respectively, as functions of the elevation angle, $\phi$. The solid line shows the radial velocity and short-dashed line presents the uniform reference velocity, which has a constant value of 1 at all times. As the elevation angle increases from $0^{\circ}$ to $30^{\circ}$, the maximum of the streamwise wind velocity component drops gradually $30 \%$, while the maximum of the vertical wind velocity components permanently increases from 0 to 0.5 . A $10 \%$ decrease in the maximum of the crosswise wind velocity component observed in the same range of the changes in elevation may be considered as insignificant. The explained variations may, however, be properly fitted by either polynomial or power series. The generated second-order polynomial fitting as applied to $U$ and $V$ and the general powerseries fitting as applied to $W$ are presented in Fig. 11 by longdashed lines. 
(a)

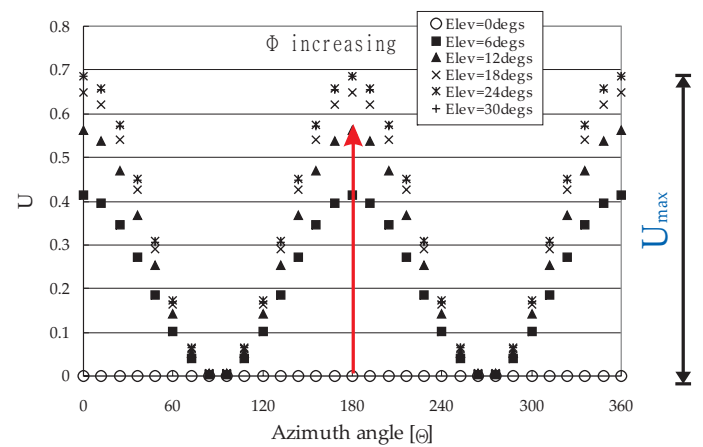

(b)

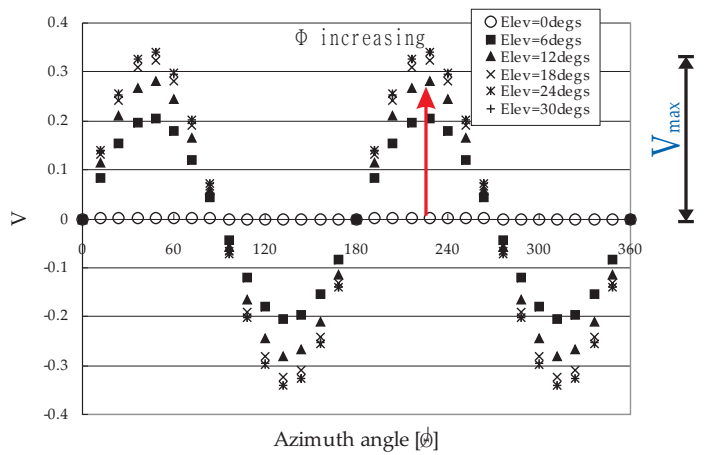

(c)

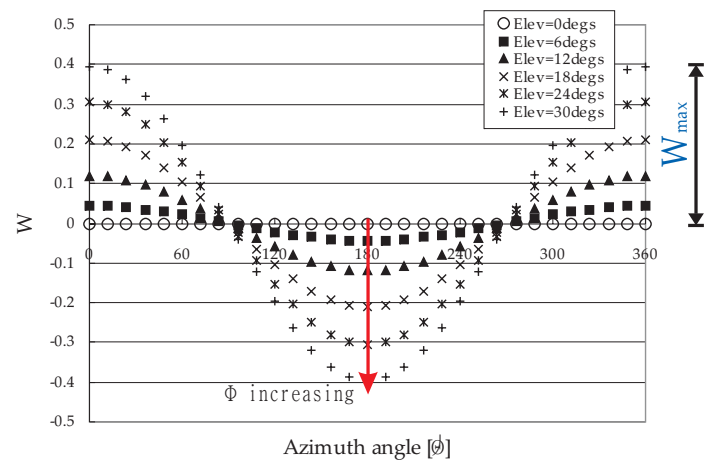

(d)

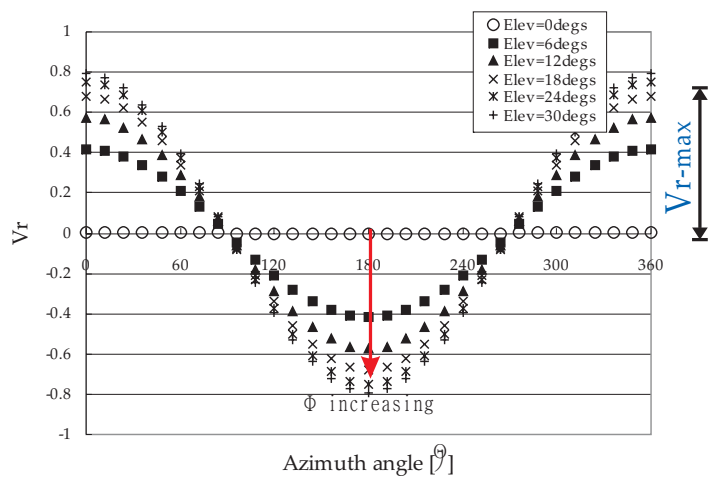

Fig. 12. Same as in Fig. 10 but for the parabolic wind velocity profile.

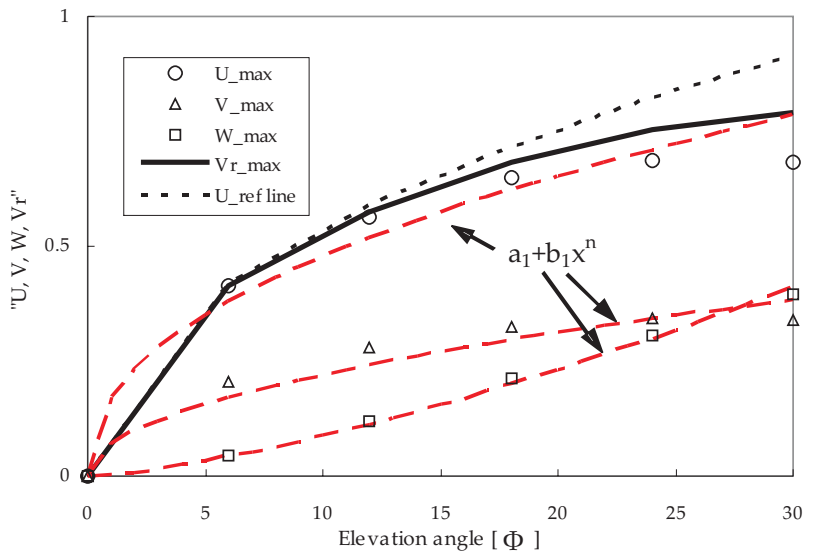

Fig. 13. Same as in Fig. 11 but for the parabolic wind velocity profile.

Figure 12 presents the streamwise, (a), crosswise, (b), and vertical, (c), wind velocity components together with radial wind velocity, (d), as functions of azimuth obtained by application of the VAD technique to the parabolic reference wind velocity distribution. In this case, as already noted above, the streamwise wind velocity component, $U$, is equal to 0 near to the ground and gradually increases up to value of 2 at the height $4 z / D=2$. In addition, as a result of the different reference wind velocity distribution, the radial velocity is relatively smaller than that in the case of the uniform reference wind velocity. A strong focus should be given here on the crucial role of the reference wind velocity distribution in accuracy of the analysis scheme of the real radar scans. It may be seen from Fig. 12 that sinusoidal variations with azimuth angle are observed in all three wind velocity components at all elevations considered. Moreover, as the elevation angle increases, the maximums of all three wind velocity components increase significantly. The directions of changes in maximums of all wind velocity components are shown by the red arrows at the first three diagrams in the figure. The maximum of the radial velocity decreases up to 0.8 as the elevation angle increases from $0^{\circ}$ to $30^{\circ}$. Figure 13 shows the changes in maximums of the calculated streamwise (circles), crosswise (triangles) and vertical (squares) wind velocity components at azimuth angles of $0^{\circ}, 45^{\circ}$ and $0^{\circ}$, respectively, as functions of the elevation angle when the parabolic wind velocity distribution is applied as a reference field. As with the case of the uniform wind velocity distribution, the increase in wind velocity with elevation may be fit by either a polynomial or power series equation. Three generated general power-series fittings as applied to $U, V$ and $W$ are presented in Fig. 13 by long-dashed lines.

Although the results presented up to now may be seen as a simple comparison with the reference wind velocity, it is clear that an important reduction in the measurement errors 


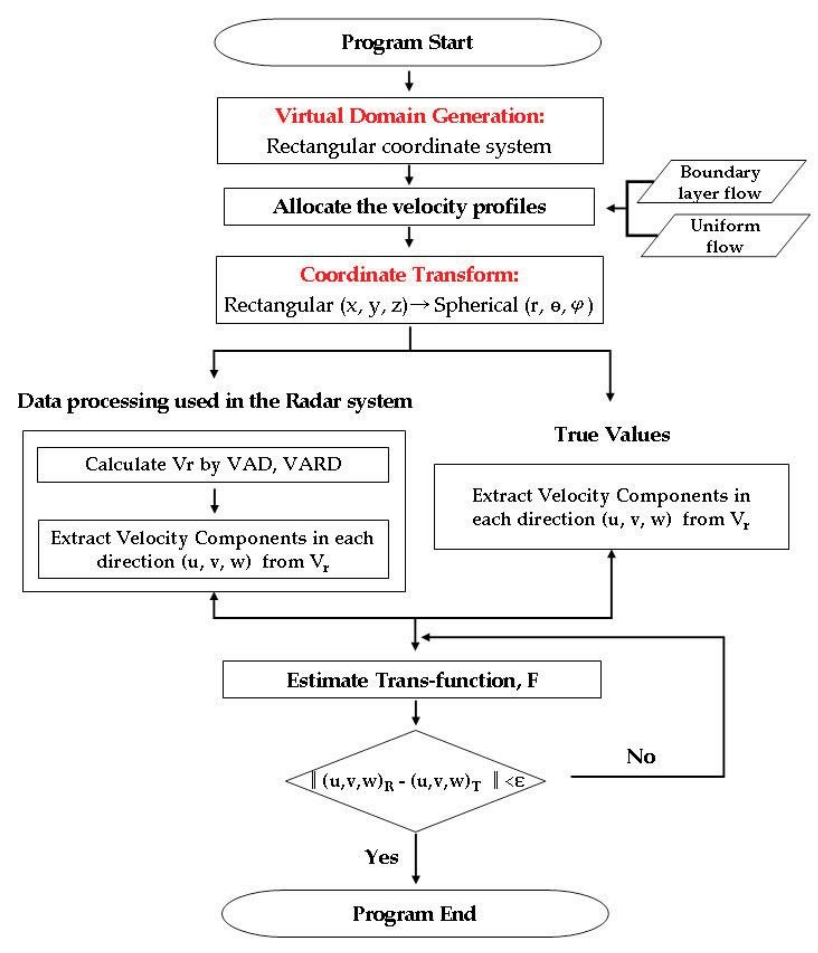

Fig. 14. Flowchart for using the trans-function fitting the transformed velocity components in spherical coordinate system.
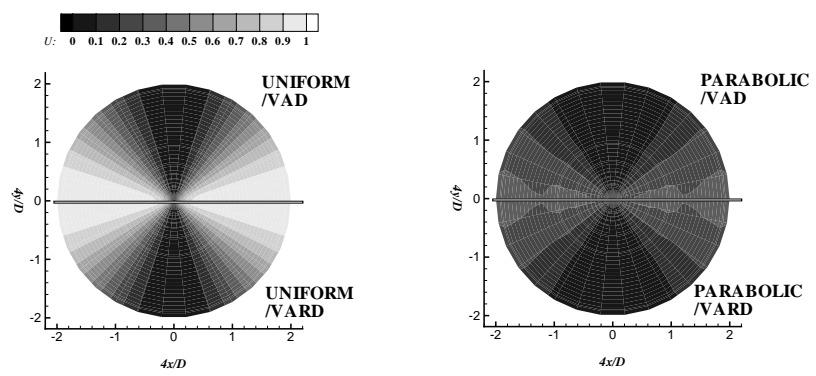

Fig. 15. Plane Position Indicator (PPI) scan at the height $4 z / D=0.1$ obtained by VAD (upper half of plot) and VARD (lower half of plot) techniques. Uniform (a) and parabolic (b) reference wind velocity distributions applied.

can be produced with the proper representation of the calculated changes in all wind velocity components. Therefore, it is logically to find a mechanism for transforming the calculated values of the wind velocity components to real preassumed value. One such ways is using transformation functions.

\subsection{Transformation function}

Transformation functions $F$ (hereafter trans-functions) may be used to transform the shape of the calculated wind velocity components $U, V$ and $W$ as obtained by using VAD/VARD
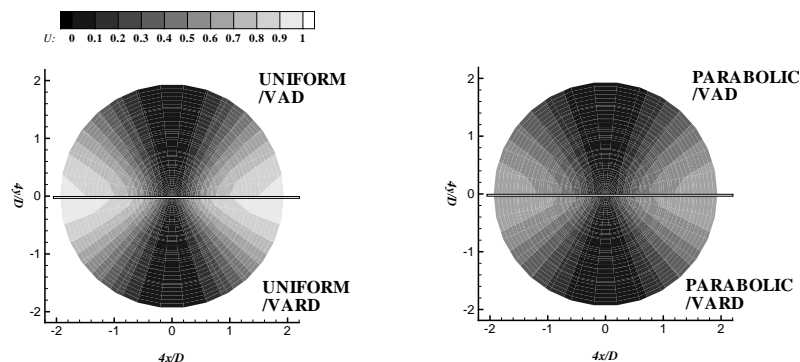

Fig. 16. Same as in Fig. 15 but at the height $4 z / D=0.5$.
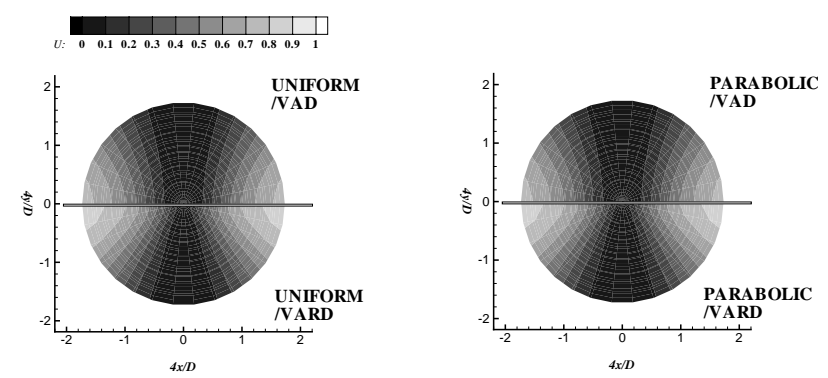

Fig. 17. Same as in Fig. 15 but at the height $4 z / D=1.0$.

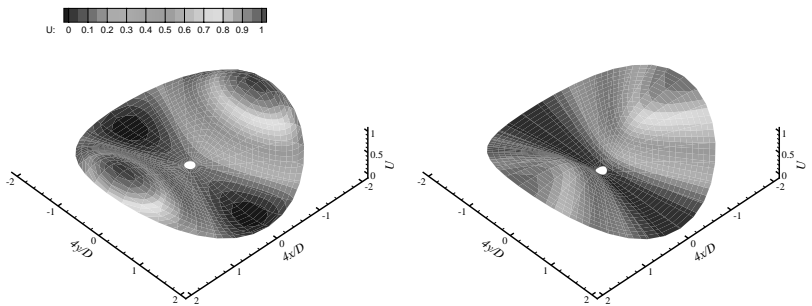

Fig. 18. Comparison of Plane Position Indicator (PPI) scan of streamwise velocity component (a) with trans-function fitted by fourth-order regression at the height $4 z / D=0.5$ and uniform reference wind velocity distribution.

techniques into the required shape in a specific pseudodomain by tracing the values of the real velocity components. The procedure which is summarized in the flow chart shown in Fig. 14 includes: (i) generation of the virtual domain and allocation of the input velocity; (ii) computation of the radial velocity by using VAD/VARD techniques; (iii) comparison of the real and transformed velocities; and, finally (iv) evaluation of the possible trans-functions reducing the errors. Note that the spherical coordinate system fixed with the radar in origin usually leads a biased interpretation of the velocity components so that the true values can be obtained indirectly by using a systematic three-dimensional curve-fitting scheme. In addition, the comparison between the flow field obtained by the trans-function and the values of real wind 


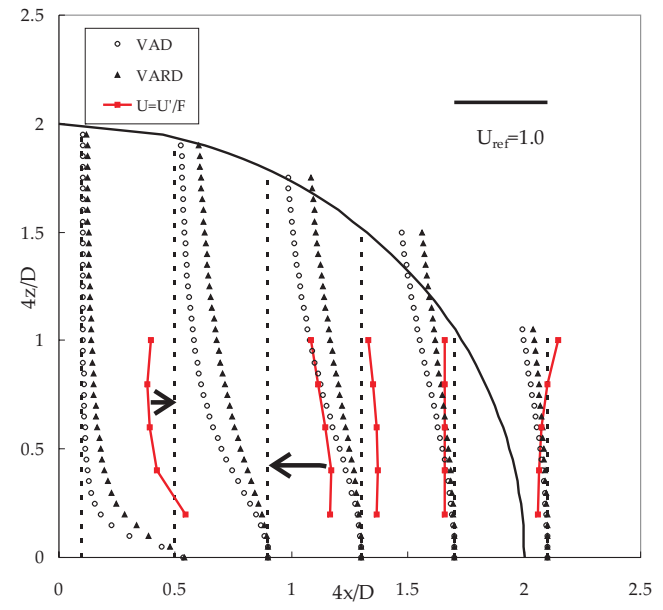

(a) 4th order regression

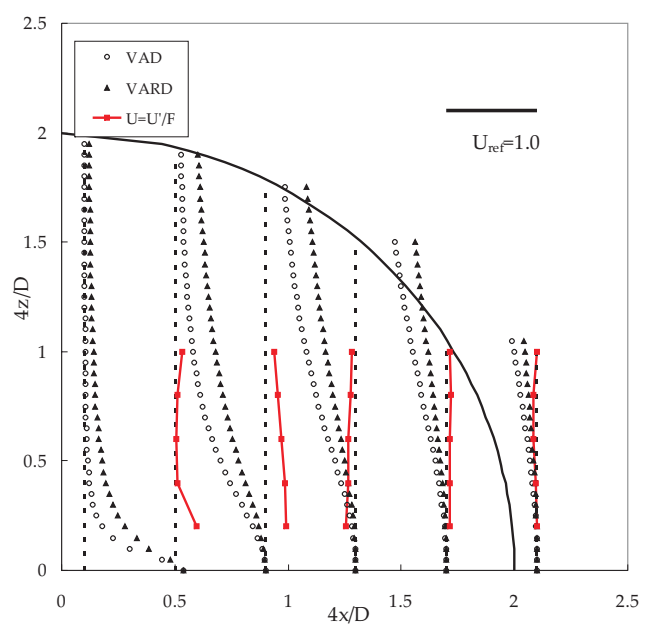

(c) 8 th order regression

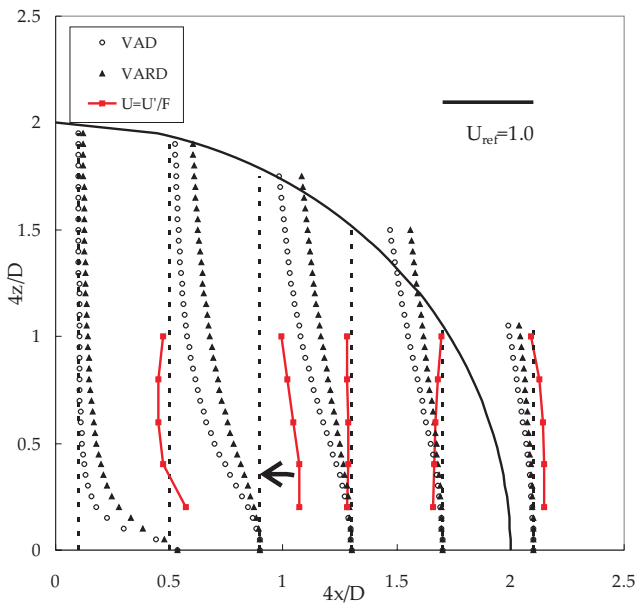

(b) 6th order regression

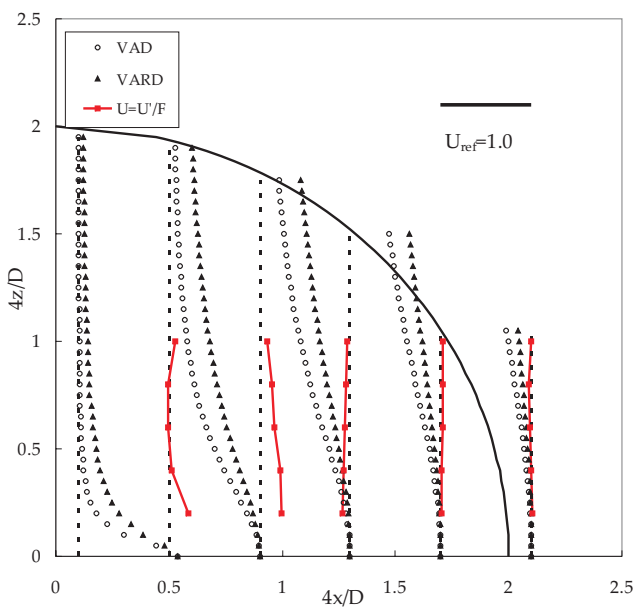

(d) 10th order regression

Fig. 19. Comparison of the profiles of streamwise velocity component along the centerline by using VARD scheme and fitted data by fourthto tenth-order regressions.

velocity may predict the error arising from the coordinate transform.

Figures 15, 16 and 17 show the contours of the streamwise wind velocity component obtained by VAD (upper half of plot) and VARD (lower half of plot) techniques to the uniform (left-hand side) and parabolic (right-hand side) reference wind velocity distributions at the heights of $4 z / D=0.1$, 0.5 and 1.0, respectively. This type of presentation is called Plane Position Indicator (PPI) plot. The direction of wind flow in the presented PPI plots is from left to right. A typical sinusoidal coordinate transform from the radial velocity may be applied to obtain the streamwise wind velocity component. In spherical coordinate system, the plane of symmetry is based on the vertical centerline so to get entire picture it would be sufficient to show only a half of the plot for the specific method and specific reference wind velocity assuming the symmetry for the second half.

As it may be seen in Fig. 15, at the height of $4 z / D=0.1$ , i.e. very close to the ground level, the streamwise velocity component predicts the reference wind velocity relatively well, remaining in the range of $10 \%$ error within the sectors covered by the azimuth ranges from $335^{\circ}$ to $25^{\circ}$ and from $155^{\circ}$ to $205^{\circ}$. Out of those ranges, however, the coincidence is very poor and gets even worse as one gets further out of the mentioned azimuth ranges. Comparing this PPI plot with the PPI plots at other heights, such as in Figs. 16 and 17, it may be concluded that the above mentioned ranges of good coincidence permanently narrows at the higher level. As compared to the VAD method, the VARD method gives relatively better result in the central domain along the vertical line. 
The streamwise wind velocity component may be fitted by a higher-order regression. For the sake of the approach, Fig. 18 presents the possibility that a reasonable agreement may be obtained between the streamwise wind velocity component and the result obtained by using trans-function of the fourth-order regression. The equivalent contours reasonably fitted by higher order regressions at the height of $4 z / D=0.5$. However, because of the geometric constraints (kind of trait) of the trans-function, the wind velocity was unrealistically overestimated at around $4 x / D=0.5$ (i.e. the second vertical solid line).

With having a help to understand the Fig. 18, the velocity contours obtained by the VAD/VARD method are compared with the contours fitted by the fourth-, sixth-, eighthand tenth-order regressions (Blane and Lei, 2000). In other words, when it is applied to the streamwise wind velocity component obtained from radar data, it is possible to improve the retrieval accuracy of the wind velocity field by these simple and effective assumptions. Figure $19 \mathrm{com}-$ pares the various profiles of the streamwise velocity component, $U$, along the centerline $4 x / D$ and shows their fitting as making a proper scaling lengthwise normalized axis $4 z / D$. Both axes are non-dimensionalized by a quarter diameter of sphere, i.e. $D / 4$, which is the characteristic length in the calculated domain. Note that the transformed wind for the reference is uniform so that all the streamwise velocity should be unity (i.e. 1) and they are all shown in dashed line. In order to regenerate the original reference wind profile, the obtained VAD and VARD profiles was transformed by the trans-function $(f)$, and it is shown by the solid-symbol line. Ideally, the solid-symbol line should be the exact solution (e.g. $\sim 1$ ), but it doesn't approach to the dashed line due to the numerical error of the regression (see the arrows). In the figure, as increasing the order of regression, the solid-symbol line approaches to the dashed line and they are gradually in good agreement. However, due to unrealistical overestimation in a region at $4 x / D=0.5$, the authors are continuing the analysis to develop the accurate wind prediction in the future.

\section{Concluding remarks}

An accurate prediction of the wind velocity fields at the virtual domain was expected to carry out (alongside) in this theoretical study. We have shown that it is possible, at a given radar domain, to regenerate the original wind velocity profile transformed by the VAD/VARD scheme and to analyze the wind flow in atmosphere around a single Doppler radar. By using the decomposition of the radial wind velocity component, authors propose the systematic approach for measuring vertical profiles of wind velocity. Two reference wind velocity distributions which modeled the real wind velocity profiles observed in nature were firstly transformed to get the directional wind speed components, while the reformulated VAD/VARD transformation equations were maintained. Then, the trans-function technique was applied to get reasonable matching between retrieved and original wind velocity components. In other words, the wind velocity components were regenerated by fitting with the higherorder regressions starting from the fourth- to tenth-order. Although no perfect agreement was found between the initial values of wind velocity components and generated fields, the streamwise wind velocity component was found to be relatively well-matched at several domains except the region of $4 x / D=0.5$, where it was over-estimated. Nevertheless, the proposed mathematical operations still produced unrealistic fields for the other wind velocity components. It is believe that the discrepancy between the calculated and initial wind fields may be overcome by using the higher order regression.

Acknowledgements. The authors wish to thank Sang-Min Jang in the Department of Atmospheric Sciences for his crucial help, PuKyong National University. This work was supported by the Korea Foundation for International Cooperation of Science \& Technology(KICOS) through a grant provided by the Korean Ministry of Science \& Technology(MOST) in 2007 (No. K2 0607010001-07A050100110).

Edited by: Zhongbo Su

\section{References}

Anderson, T.: VAD winds from C band Ericsson Doppler Weather Radars, Meteor. Z., 7, 309-319, 1998.

Armstrong, G. and Donaldson Jr., R.: Plan shear indicator for realtime Doppler radar identification of hazardous storms, J. Appl. Meteor., 8, 376-383, 1969.

Blane, M. and Lei, Z.: The 3L Algorithm for Fitting Implicit Polynomial Curves and Surfaces to Data, IEEE Transactions on Pattern Analysis and Machine Intelligence, 22, 298-313, 2000.

Browning, K. and Wexler, R.: The determination of kinematic properties of a wind field using Doppler radar, J. Appl. Meteor., 7, 105-113, 1968.

Caton, P.: The measurement of wind and convergence by Doppler Radar, in: Preprints 10th Weather Radar Conference, 1963.

Easterbrook, C.: Estimating Horizontal Wind Fields by Two Dimensional Curve Fitting of Single Doppler Radar Measurements, in: Preprints 16th Radar Meteorology Conference, 1975.

Lhermitte, R. and Atlas, D.: Precipitation motion by pulse Doppler radar, in: Preprints 9th Conf. on Radar Meteorology, 1961.

Matejka, T.: Concurrent extended vertical velocity azimuth display (CEVAD), in: Preprints 26th Conf. on Radar Meteorology, 1993.

Srivastava, R., Matejka, T., and Lorello, T.: Doppler radar study of the trailing anvil region associated with a squall line, J. Atmos. Sci., 43, 356-377, 1986.

Waldteufel, P. and Corbin, H.: On the analysis of single-Doppler radar data, J. Appl. Meteorol., 18, 532-542, 1978.

Wood, V. and Brown, R.: Single-Doppler velocity signature interpretation of non-divergent environmental winds, J. Atmos. Ocean. Technol., 3, 114-128, 1986.

Wood, V. and Brown, R.: Effects of radar sampling on singleDoppler velocity signatures of mesocyclones and tornadoes., Wea. Forecast., 12, 928-938, 1997. 